\title{
NAMPT protects against 6-hydroxydopamine-induced neurotoxicity in PC12 cells through modulating SIRT1 activity
}

\author{
XIAO-DONG ZOU ${ }^{1 *}$, SHAO-QING GUO ${ }^{2 *}$, ZHI-WEI HU ${ }^{1}$ and WEI-LANG LI ${ }^{1}$ \\ ${ }^{1}$ Department of Neurology, Tongde Hospital of Zhejiang Province; ${ }^{2}$ Department of Massage, \\ The Third Affiliated Hospital of Zhejiang Province Traditional Chinese Medical University, \\ Hangzhou, Zhejiang 310012, P.R. China
}

Received December 1, 2014; Accepted January 25, 2016

DOI: $10.3892 / \mathrm{mmr} .2016 .5034$

\begin{abstract}
Parkinson's disease (PD) is the second most common progressive neurodegenerative movement disorder. Nicotinamide phosphoribosyltransferase (NAMPT) catalyzes the first rate-limiting step in the nicotinamide adenine dinucleotide $\left(\mathrm{NAD}^{+}\right)$biosynthetic pathway in mammals, is a substrate for $\mathrm{NAD}^{+}$-dependent enzymes, such as sirtuin 1 (SIRT1), and contributes to cell fate decisions. However, the role of NAMPT in PD has remained to be fully elucidated. In the present study, PC12 cells were treated with the neurotoxin 6-hydroxydopamine (6-OHDA) to establish an in vitro model of $\mathrm{PD}$, following which an obvious inhibitory effect on the levels of NAMPT and $\mathrm{NAD}^{+}$as well as the $\mathrm{NAD}^{+} / \mathrm{NADH}$ ratio was detected. In addition, pre-incubation with FK866, a highly specific NAMPT inhibitor, enhanced the inhibitory effects of 6-OHDA on the viability of PC12, while pre-incubation with nicotinamide mononucleotide (NMN), am enzymatic product of NAMPT, had the opposite effect. Furthermore, it was revealed that NMN markedly attenuated 6-OHDA-induced decreases in superoxide dismutase activity and glutathione levels, as well as 6-OHDA-induced increases in malondialdehyde and lactate dehydrogenase in PC12 cells. Furthermore, 6-OHDA significantly reduced SIRT1 activity in PC12 cells, which was inhibited by NMN. The pharmacological activator resveratrol also significantly inhibited 6-OHDA-mediated decreases in PC12 cell viability while reversing 6-OHDA-induced decreases in SIRT1 levels. The results of the present study suggested that NMT protected against 6-OHDA-induced decreases in PC12 cell viability, and that SIRT1 activation had a role in this process. Treatment with
\end{abstract}

Correspondence to: Dr Xiao-Dong Zou, Department of Neurology, Tongde Hospital of Zhejiang Province, 234 Gucui Road, Xihu, Hangzhou, Zhejiang 310012, P.R. China

E-mail: zou_xiaodong12@163.com

*Contributed equally

Key words: nicotinamide phosphoribosyltransferase, PC12 cells, 6-hydroxydopamine, nicotinamide adenine dinucleotide, sirtuin 1
NMN to activate SIRT1 may represent a novel therapeutic strategy for treating PD.

\section{Introduction}

Parkinson's disease (PD), a chronic and progressive neurodegenerative movement disorder, is characterized by progressive degeneration of dopaminergic neurons in the substantia nigra pars compacta (SNc) (1). The mechanisms of dopaminergic cell death have remained to be fully elucidated. Lipid peroxidation, oxidative stress and mitochondrial dysfunction are considered to mediate the progression of dopaminergic neuron degeneration (2). As the etiology and pathogenesis of PD have remained elusive, current therapies focus on the symptoms, rather than the neurodegenerative progression of dopaminergic neurons in SNc. Therefore, novel insight into the disease mechanisms is urgently required. 6-Hydroxydopamine (6-OHDA), which has been previously used to establish in vivo as well as in vitro models of $\mathrm{PD}$, is a neurotoxin mainly targeting dopaminergic neurons $(3,4)$. 6-OHDA exerts its effects via inducing reactive oxygen species (ROS) overproduction and energy depletion $(1,5)$. PC12 cells are widely used for in vitro studies exploring the mechanisms of neurodegenerative diseases (6-8).

Seven members of the sirtuin family have been associated with energy production, cell metabolism and DNA repair in mammals. They are deacetylases acting on histones in the presence of nicotinamide adenine dinucleotide $\left(\mathrm{NAD}^{+}\right)(9)$. Nicotinamide phosphoribosyltransferase (NAMPT) is the rate-limiting enzyme in the mammalian $\mathrm{NAD}^{+}$salvage pathway for the conversion of nicotinamide into nicotinamide mononucleotide (NMN), which is later converted to $\mathrm{NAD}^{+}(10)$. NAMPT is thought to function in a manner equivalent to that of pyrazinamidase/nicotinamidase 1 in mammals (11), and increased expression of NAMPT has been shown to positively regulate $\mathrm{NAD}^{+}$levels and enhance SIRT1 transcriptional regulatory activity in mouse fibroblasts (12). The $\mathrm{NAD}^{+} / \mathrm{NADH}$ ratio is a fundamental indicator of the cellular redox status (13) and ROS accumulation has been shown to modify the activity of sirtuins (14). Furthermore, a previous study demonstrated that NMN protected the rotenone-induced PC12 cells from cell death by restoring the intracellular levels of $\mathrm{NAD}^{+}$and preventing ATP depletion (15). 
SIRT1 has beneficial effects on numerous major aging-associated pathologies, including diabetes (16), neurodegeneration (17), chronic heart failure (18) and cancer (19). Resveratrol treatment was shown to attenuate cell stress caused by to caloric restriction via activation of SIRT1 (20). SIRT1 also regulates an array of transcription factors, including nuclear factor $\kappa \mathrm{B}(21)$ and p53 (22). High levels of ROS induce the expression of SIRT1 (23), which in turn initiates DNA repair (24).

In the present study, the neuroprotective effects of the NAMPT metabolite NAD ${ }^{+}$against 6-OHDA-induced neurotoxicity in PC12 cells were elucidated. Furthermore, the possible underlying mechanisms were examined by using an NAMPT inhibitor and by assessing effects on indicators of oxidative damage as well as SIRT1 activation.

\section{Materials and methods}

Cell culture and treatment. The PC12 rat adrenal pheochromocytoma cell line was obtained from the Type Culture Collection of the Chinese Academy of Sciences (Shanghai, China). PC12 cells were maintained in Dulbecco's modified Eagle's medium supplemented with $5 \%$ (v/v) fetal bovine serum (Invitrogen; Thermo Fisher Scientific, Inc., Waltham, MA, USA), $10 \%$ heat-inactivated equine serum (Hyclone, Logan, UT, USA), $100 \mathrm{U} / \mathrm{ml}$ penicillin and $0.1 \mathrm{mg} / \mathrm{ml}$ streptomycin (Sigma-Aldrich, St Louis, MO, USA). Cells were incubated at $37^{\circ} \mathrm{C}$ in a humidified atmosphere with $5 \% \mathrm{CO}_{2}$. The medium was replaced every two or three days and cells were routinely subcultured at a 1:5 ratio at weekly intervals. For the experiments, PC12 cells were pre-incubated with NMN $(600 \mu \mathrm{M})$, FK866 $(10 \mathrm{~nm})$ or resveratrol $(50 \mu \mathrm{M}$; Sigma-Aldrich) for $2 \mathrm{~h}$ and then exposed to 6-OHDA for $24 \mathrm{~h}$. The control group was treated with an equivalent volume of dimethyl sulfoxide (DMSO; final concentration, 0.1\%; Sigma-Aldrich) added to the medium.

Cell viability assay. Cell viability was estimated using a 3-(4,5-dimethylthiazol-2-yl)-2,5-diphenyltetrazolium bromide (MTT) assay. In brief, PC12 cells were seeded in 96-well plates at 10,000 cells/well for $24 \mathrm{~h}$. After 6-OHDA treatment for $24 \mathrm{~h}$ with or without pre-incubation with NMN, FK866 or resveratrol, the cells were incubated with $20 \mu \mathrm{l}$ MTT solution ( $5 \mathrm{mg} / \mathrm{ml}$; Sigma-Aldrich) for $4 \mathrm{~h}$. The culture medium was removed and the dark blue formazan product was dissolved by adding $150 \mu \mathrm{l}$ DMSO to each well. The absorbance of each well was read at $570 \mathrm{~nm}$ using the Rayto-RT 6000 enzyme-linked immunosorbent assay reader (Rayto Life and Analytical Sciences, Guangdong, China), and the viability was expressed as the percentage of the untreated cells.

$N A D^{+} / N A D H$ and SIRT1 activity assays. An $\mathrm{NAD}^{+} / \mathrm{NADH}$ quantification kit was used to determine $\mathrm{NAD}^{+}$and $\mathrm{NADH}$ levels (BioVision, Mountain View, CA, USA) according to the manufacturer's instructions. To assess SIRT1 activity, whole-cell extracts were prepared using a mild lysis buffer plus protease inhibitor cocktail (Roche Diagnostics, Basel, Switzerland). An Infinite M200 microplate fluorimeter (Tecan, Hillsborough, NC, USA) was used to measure SIRT1 activity.
Assessment of glutathione (GSH) and malondialdehyde (MDA) levels. PC12 cells were washed with $1 \mathrm{ml}$ phosphate-buffered saline (PBS) three times and subsequently scraped off the bottom of the flask in ice-cold PBS. Lysis buffer was then added to the cells, followed by incubation for $40 \mathrm{~min}$ on ice. The lysates were centrifuged at $10,000 \mathrm{x} \mathrm{g}$ for $10 \mathrm{~min}$ at $4^{\circ} \mathrm{C}$. The supernatant was collected and the protein content was determined using the bicinchoninic acid (BCA) protein assay kit, followed by analysis of the GSH and MDA content using a commercial colorimetric GSH and MDA assay kit (Beyotime Institute of Biotechnology, Haimen, China).

Assessment of superoxide dismutase (SOD) activity and lactate dehydrogenase $(\mathrm{LDH})$. According to the manufacturer's instructions, $100 \mu \mathrm{l}$ culture supernatant was used to determine SOD activity using a commercially available detection kit (Nanjing Jiancheng Biochemical Reagent Co., Nanjing, China). For the LDH assay, $80 \mu \mathrm{l}$ culture supernatant was collected and subjected to analysis using an LDH assay kit (Nanjing KeyGen Biotech. Co., Ltd., Nanjing, China) according to the manufacturer's instructions.

Western blot analysis. Western blot analysis was performed according to the protocol of a previous study (25). PC12 cells $\left(10^{6}\right.$ cells/well) were seeded onto six-well plates and treated with 6-OHDA for $24 \mathrm{~h}$ with or without pre-incubation with FK866, NMN or resveratrol. Subsequently, cells were lysed in radioimmunoprecipitation assay buffer (Beyotime Institute of Biotechnology) containing protease inhibitor cocktail (Roche Diagnostics) and then incubated on ice for $30 \mathrm{~min}$. The cellular debris was removed by centrifugation $(14,000 \mathrm{x} \mathrm{g}$ for $10 \mathrm{~min}$ at $4^{\circ} \mathrm{C}$ ) and the protein concentration in the supernatant was determined using a BCA protein assay kit. Equal amounts of protein (20-30 $\mu \mathrm{g}$ ) were subjected to $10 \%$ sodium dodecyl sulfate polyacrylamide gel electrophoresis and transferred onto a polyvinylidene difluoride membrane (Bio-Rad Laboratories, Inc., Hercules, CA, USA). After blocking in 5\% non-fat milk for $1 \mathrm{~h}$ at room temperature, blots were incubated with rabbit monoclonal anti-NAMPT $(1: 1,000$; Abcam, Cambridge, MA, USA; cat. nos. ab45890), anti-SIRT1 antibody $(1: 1,000$; cat. no. ab32441) and mouse monoclonal $\beta$-actin (1:1,000; cat. no. ab6276) overnight at $4^{\circ} \mathrm{C}$. Subsequent to three washes in Tris-buffered saline containing Tween 20, membranes were incubated with goat anti-rabbit horseradish peroxidase-conjugated IgG or goat anti-mouse horseradish peroxidase-conjugated IgG (1:5,000; Beyotime Institute of Biotechnology; cat. nos. A0208 and A0216, respectively) for $1 \mathrm{~h}$ at room temperature. Proteins were detected using the enhanced chemiluminescence western blot detection kit (Amersham ECL plus Western blotting detection system; GE Healthcare). The blots were washed again and scanned. The proteins of interest were detected using the Odyssey Western Detection system and quantified with the Odyssey LI-COR System software (LI-COR Biosciences, Lincoln, NE, USA).

Statistical analysis. Experiments were repeated at least three times with consistent results. Values are expressed as the mean \pm standard error of the mean and one-way analysis of variance followed by Tukey's multiple comparisons test was 
A

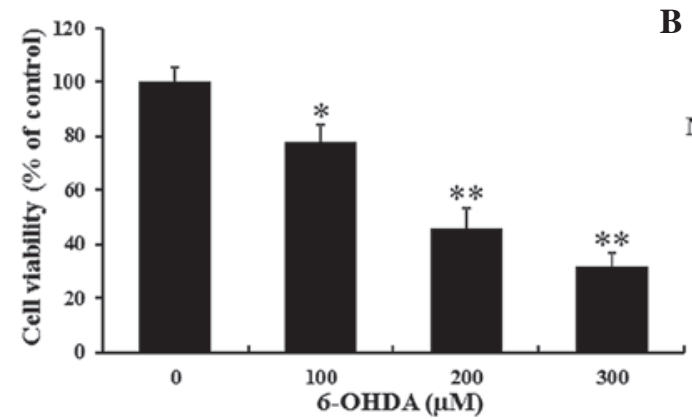

B

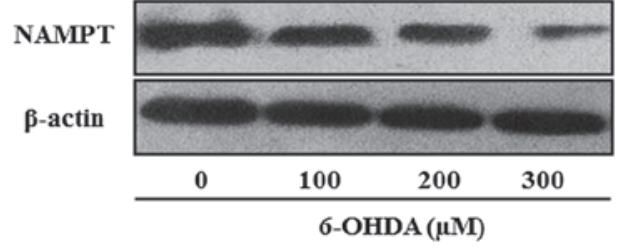

Figure 1. Effects of 6-OHDA treatment on the viability of PC12 cells and expression of NAMPT. PC12 cells were incubated with 6-OHDA (0, 100, 200 and $300 \mu \mathrm{M}$ ) for $24 \mathrm{~h}$. (A) MTT assay revealed that 6-OHDA dose-dependently decreased cell viability. Values are expressed as the mean \pm standard error $(\mathrm{n}=3)$. ${ }^{*} \mathrm{P}<0.05$ and ${ }^{* * *} \mathrm{P}<0.01$ vs. the control group. (B) Western blot analysis revealed that the expression of NAMPT in PC12 cells is downregulated by 6-OHDA in a dose-dependent manner. NAMPT, nicotinamide phosphoribosyltransferase; 6-OHDA, 6-hydroxydopamine.
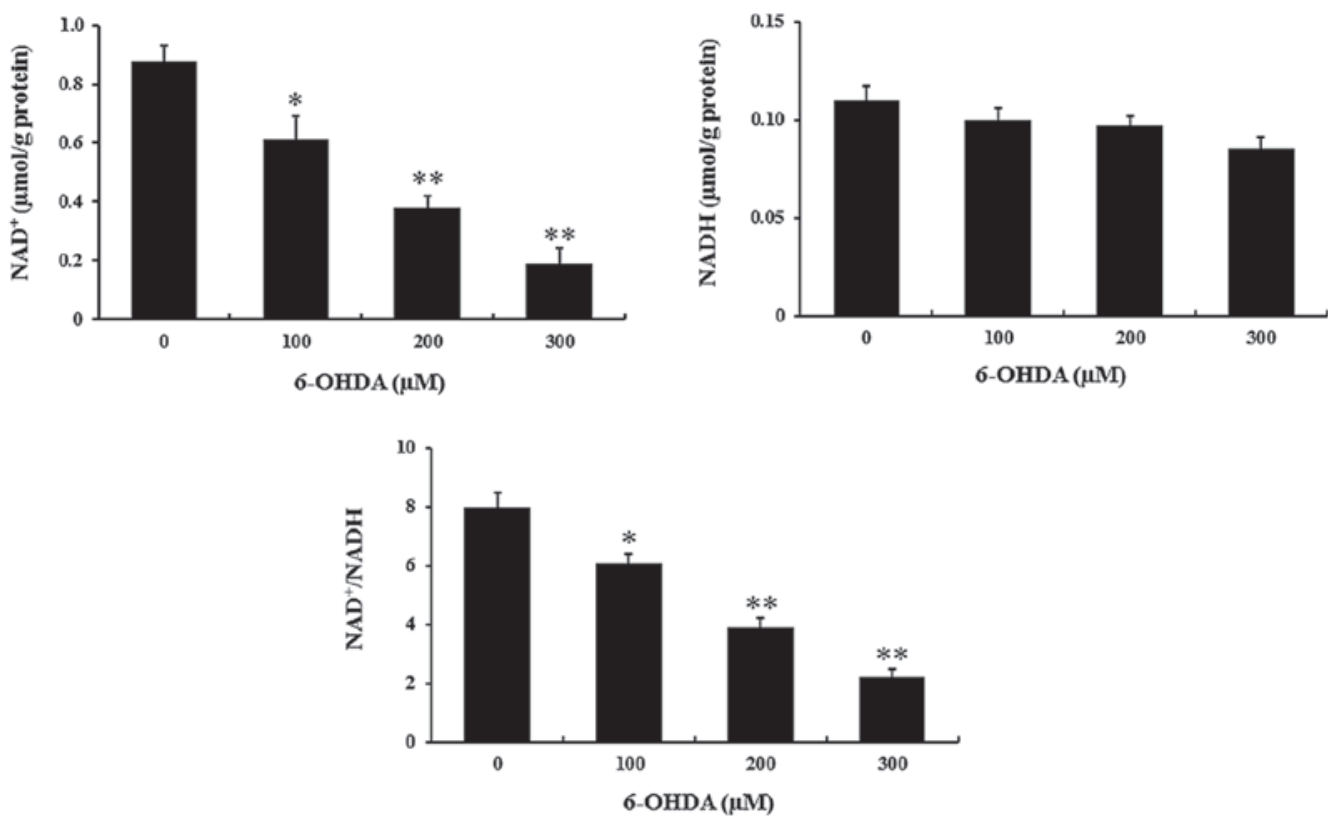

Figure 2. 6-OHDA dose-dependently decreases the levels of $\mathrm{NAD}^{+}$and the $\mathrm{NAD}^{+} / \mathrm{NADH}$ ratio in PC12 following treatment for $24 \mathrm{~h}$. Values are expressed as the mean \pm standard error of the mean $(n=4)$. ${ }^{*} \mathrm{P}<0.05$ and ${ }^{* *} \mathrm{P}<0.01$ vs. the control group. 6-OHDA, 6-hydroxydopamine; NAD, nicotinamide adenine dinucleotide.

used for comparisons between multiple groups. Statistical analysis was performed using GraphPad Prism 6.0 (GraphPad Software, La Jolla, CA, USA). P $<0.05$ was considered to indicate a statistically significant difference.

\section{Results}

6-OHDA-induced decreases in PC12 cell viability are accompanied with a reduction in NAMPT and NAD levels. According to the protocols of previous studies $(8,26)$, the present study generated a 6-OHDA-induced in vitro model of neurodegeneration resembling PD. PC12 cells were treated with various concentrations of 6-OHDA for $24 \mathrm{~h}$ and the cell viability was determined using an MTT assay. In accordance with the findings of the previous studies, the viability of $\mathrm{PC} 12$ cells was decreased by 6-OHDA in a dose-dependent manner. Only $\sim 50 \%$ of PC12 cells survived after exposure to $200 \mu \mathrm{M}$ 6-OHDA for $24 \mathrm{~h}$ (Fig. 1A).
To investigate the effects of 6-OHDA-induced neuronal cell death on NAMPT levels in PC12 cells, western blot analysis was performed. Compared with the control group, NAMPT expression was markedly decreased by 6-OHDA in a dose-dependent manner (Fig. 1B).

As studies have indicated that $\mathrm{NAD}^{+}$levels are regulated by NAMPT in mammalian neurons, the present study detected the concentration of $\mathrm{NAD}^{+}$and $\mathrm{NADH}$ in response to 6-OHDA treatment. Compared with that in the control group, the $\mathrm{NAD}^{+}$release was significantly decreased by 6-OHDA treatment $(\mathrm{P}<0.01)$ (Fig. 2), while NADH levels were not affected. Consequently, the $\mathrm{NAD}^{+} / \mathrm{NADH}$ ratio was reduced by 6-OHDA in a dose-dependent manner $(\mathrm{P}<0.01)$ (Fig. 2).

NMN protects $P C 12$ cells against 6-OHDA-induced death, while NAMPT inhibition aggravates 6-OHDA-induced PC12 cell death. In order to determine the roles of NAMPT in the molecular pathology of PD, PC12 cells were 
A

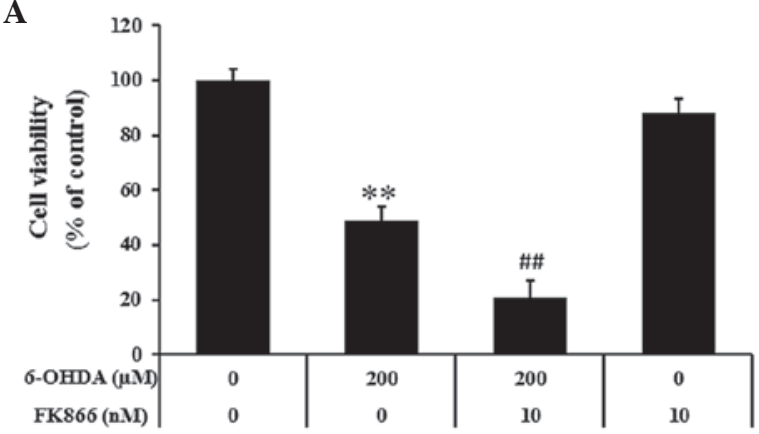

B

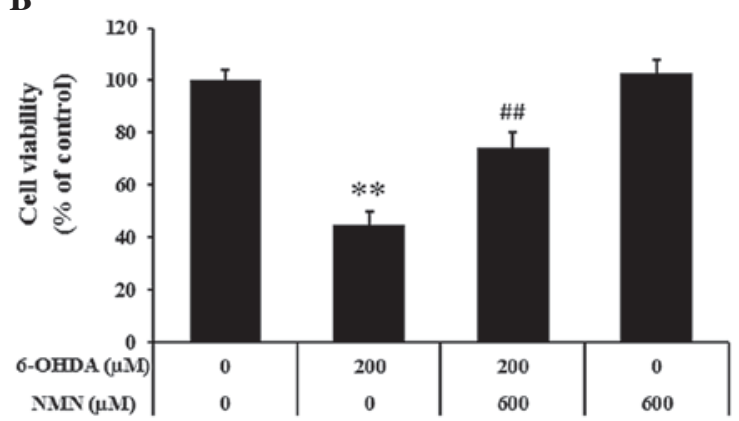

Figure 3. FK866 enhances, while NMN attenuates the inhibitory effects of 6-OHDA on PC12 cell viablilty. (A) PC12 cells were pre-treated with nicotinamide phosphoribosyltransferase inhibitor FK866 for $1 \mathrm{~h}$, followed by incubation with $200 \mu \mathrm{M} 6$-OHDA for $24 \mathrm{~h}$. (B) PC12 cells were incubated with $200 \mu \mathrm{M}$ 6-OHDA for $24 \mathrm{~h}$ after pre-treatment with $\mathrm{NMN}$ for $1 \mathrm{~h}$. Values are expressed as the mean \pm standard error $\left(\mathrm{n}=3\right.$ ). ${ }^{* *} \mathrm{P}<0.01$ vs. control group; ${ }^{* \#} \mathrm{P}<0.01 \mathrm{vs}$. 6-OHDA group. 6-OHDA, 6-hydroxydopamine; NMN, nicotinamide mononucleotide.

A

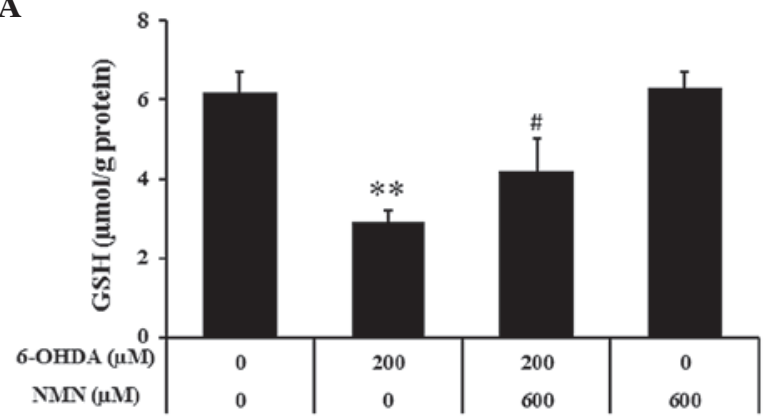

C

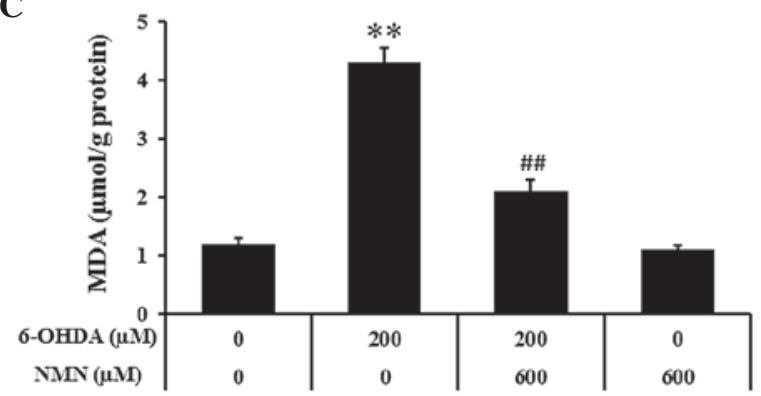

B

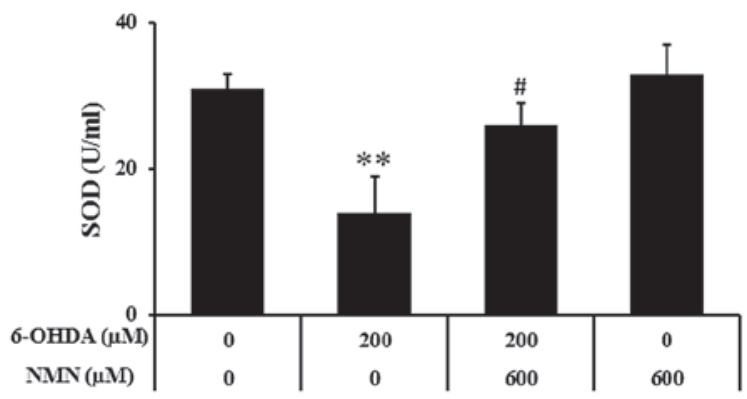

D

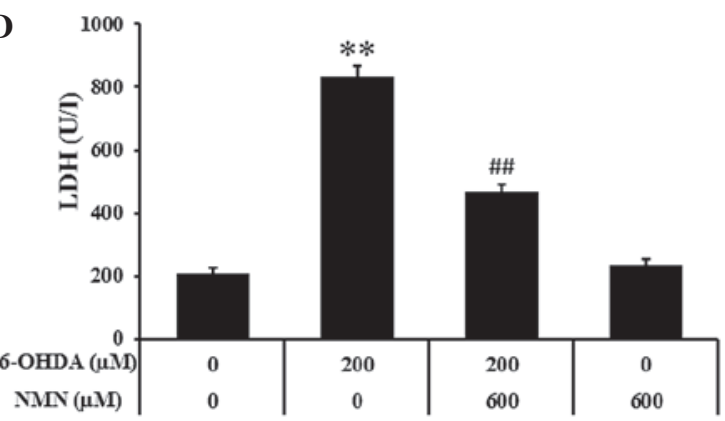

Figure 4. Pre-treatment with NMN prevents 6-OHDA-induced oxidative damage and LDH release by PC12 cells. Effects of 6-OHDA and/or NMN on (A) GSH, (B) SOD, (C) MDA and (D) LDH levels in PC12 cells after treatment with 6-OHDA for 24 h. Values are expressed as the mean \pm standard error of the mean $(\mathrm{n}=3) .{ }^{* *} \mathrm{P}<0.01$ vs. control group; ${ }^{\#} \mathrm{P}<0.05,{ }^{\# \#} \mathrm{P}<0.01$ vs. 6-OHDA group. SOD, superoxide dismutase; LDH, lactate dehydrogenase; GSH, glutathione; NMN, nicotinamide mononucleotide; MDA, malondialdehyde; 6-OHDA, 6-hydroxydopamine.

pre-treated with FK866, a NAMPT-specific inhibitor, or NMN, the enzymatic product of NAMPT, prior to induction with 6-OHDA. As shown in Fig. 3A, pre-incubation with FK866 $(10 \mathrm{nM})$ significantly decreased the viability of PC12 cells following treatment with 6-OHDA from $\sim 50$ to $21 \%(\mathrm{P}<0.01)$. Furthermore, $\mathrm{PC} 12$ cells were pre-incubated with NMN $(600 \mu \mathrm{M})$ followed by incubation with 6-OHDA $(200 \mu \mathrm{M})$ for $24 \mathrm{~h}$ (Fig. 3B). It was observed that NMN markedly increased the viability of 6-OHDA-treated PC12 cells from $\sim 45$ to $74 \%$ ( $\mathrm{P}<0.01)$. These results demonstrated that following induction of PC12 cells with 6-OHDA, prior inhibition of NAMPT activity decreased the cell viability, while NMN, the enzymatic product of NAMPT, rescued cell viability.
NMN prevents 6-OHDA-induced oxidative damage of PC12 cells and LDH release. Previous studies have demonstrated that oxidative stress is as an important mediator in 6-OHDA-induced cell death $(27,28)$. To explore whether NMN protects PC12 cells against 6-OHDA induced cell death due to its anti-oxidant capacity, the levels of GSH and SOD, which are the most common indicators of anti-oxidant activity, were assessed in PC12 cells. As a vital enzymatic anti-oxidant, SOD has an important role in the clearance of ROS. As shown in Fig 4A and B, treatment with $200 \mu \mathrm{M}$ 6-OHDA significantly reduced the intracellular concentration of GSH and SOD, which was partially inhibited by treatment with $600 \mu \mathrm{M}$ NMN $(\mathrm{P}<0.05)$. Furthermore, the concentration of MDA, a lipid oxidation biomarker, was assessed. After incubation 
A

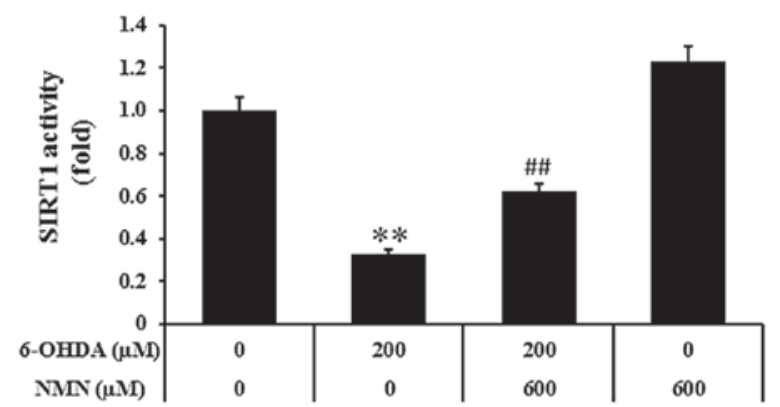

B

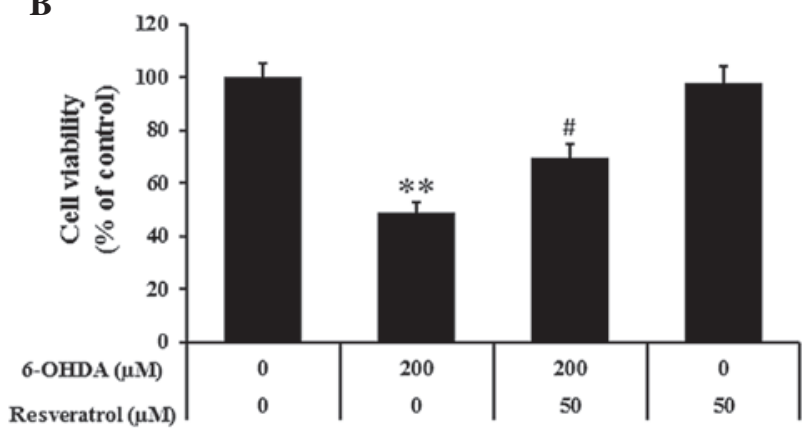

C

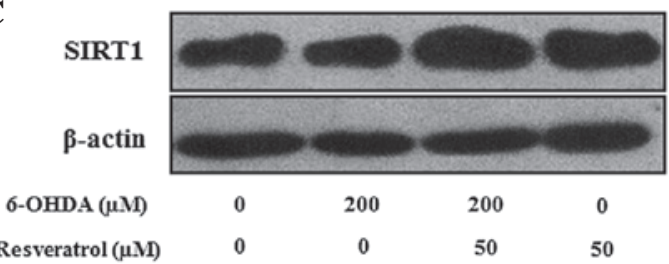

Figure 5. NMN and resveratrol may attenuate 6-OHDA-induced decreases in cell viability via enhancing SIRT1 activity. (A) Pre-treatment with NMN enhances SIRT1 activity in PC12 cells after treatment with 6-OHDA for 24 h. (B) Resveratrol reduces the inhibitory effects of 6-OHDA on PC12 cell viability. Values are expressed as the mean \pm standard error of the mean $(\mathrm{n}=3)$. ${ }^{* *} \mathrm{P}<0.01$ vs. the control group; ${ }^{\#} \mathrm{P}<0.05$ and ${ }^{\# \#} \mathrm{P}<0.01$ vs. the 6 -OHDA group. (C) Pre-treatment with resveratrol enhances SIRT1 activity in PC12 cells after treatment with 6-OHDA for 24 h. SIRT, sirtuin; NMN, nicotinamide mononucleotide; 6-OHDA, 6-hydroxydopamine.

with 6-OHDA for $24 \mathrm{~h}$, the levels of MDA were significantly increased $(\mathrm{P}<0.01)$, which was attenuated by NMN $(600 \mu \mathrm{M})$ $(\mathrm{P}<0.01)$ (Fig. 4C). The release of LDH into the culture medium due to plasma membrane damage is an indicator of cell death. As shown in Fig. 4D, 6-OHDA markedly enhanced LDH release, which was significantly attenuated by NMN $(\mathrm{P}<0.01)$. All of these results indicated that NMN protects PC12 cells from 6-OHDA-induced death due to its anti-oxidant activity.

NMN may exert its neuroprotective effects by activating SIRT1. Since SIRT1 is known to mediate several biological effects of NAMPT, the present study further assessed whether the protective effects of NMN against 6-OHDA-induced PC12 cell death may be mediated via modulation of SIRT1 activity. Indeed, the results indicated that 6-OHDA markedly decreased the activity of SIRT1 compared to that in the control group, while NMN attenuated this effect $(\mathrm{P}<0.01)$ (Fig. 5A). The pharmacological activator resveratrol is known to exert protective effects on PC12 cells against 6-OHDA-induced toxicity. Pre-treatment with resveratrol $(50 \mu \mathrm{M})$ markedly reduced the inhibitory effects of 6-OHDA on cell viability $(\mathrm{P}<0.05)$ (Fig. 5B). Furthermore, western blot analysis showed that the 6-OHDA-induced reduction of SIRT1 expression was reversed by pre-treatment with resveratrol (Fig. 5C). All of these results indicated that enhanced SIRT1 activity and increased SIRT1 expression are associated with the protective effects of NMN against 6-OHDA-induced PC12 cell death.

\section{Discussion}

The present study demonstrated that NMN, the enzymatic product of NAMPT, exerts protective effects against 6-OHDA-induced neurodegeneration in vitro. It was revealed that following 6-OHDA treatment, $\mathrm{PC} 12$ cell viability was decreased, NAMPT was downregulated, and the levels of $\mathrm{NAD}^{+}$as well as the $\mathrm{NAD}^{+} / \mathrm{NADH}$ ratio were significantly decreased. Indicators of cell death and oxidative damage were increased, and SIRT1 activation was decreased following treatment with 6-OHDA, which was attenuated by treatment with NMN. Furthermore, the NAMPT inhibitor FK866 was shown to aggravate the cytotoxic effects of 6-OHDA. The present study therefore indicated that decreases in NAMPT/NAD+ and subsequent deactivation of SIRT1 may be implicated in the molecular pathology of neurodegenerative disorders, including PD.

The neurotoxin 6-OHDA induces dopaminergic neuronal death and has been widely used to establish in vitro models of PD (1). 6-OHDA exerts its toxic effects via inducing ROS overproduction, which results in oxidative stress and cell death, through the following pathways: Extracellular auto-oxidation, monoamine oxidase-mediated intracellular metabolism and mitochondrial respiratory chain inhibition $(29,30)$. PC12 cells have been widely used in studies examining the molecular mechanisms of neurodegenerative diseases $(6,8)$. Therefore, 6-OHDA-treated PC12 cells were used in the present study as a cell model to investigate the implication of NAMPT in PD.

In the present study, 6-OHDA was shown to concentration-dependently reduce $\mathrm{NAD}^{+}$levels; this may have been a consequence of the simultaneously observed reduction of NAMPT, as NAMPT is required for the production of $\mathrm{NAD}^{+}$(31). While NADH levels were not affected by 6-OHDA, the $\mathrm{NAD}^{+} / \mathrm{NADH}$ ratio was decreased, which may explain for the observed reduced cell survival rate due to various stressors (32). In addition, increases in NAMPT or NMN have been previously indicated to markedly reduce cell death (31), which was consistent with the observations of the present study that NMN enhanced the survival of PC12 cells after incubation with 6-OHDA. Furthermore, pre-incubation 
with the NAMPT inhibitor FK866 aggravated the cytotoxic effects of 6-OHDA, which further suggested the neuroprotective effects of NAMPT/NMN.

In neurodegenerative disorders, oxidative stress originates from ROS overproduction and impaired anti-oxidative defense is a major reason of neuronal death (33). The antioxidant defense system consists of non-enzymatic anti-oxidants such as GSH and enzymes such as SOD, which neutralize free radicals. The present study revealed that, compared with those in the control group, the levels of GSH and SOD were markedly reduced in 6-OHDA-treated PC12 cells, which was attenuated by pre-treatment with NMN. These results suggested that NMN, an enzymatic product of NAMPT, attenuated the 6-OHDA-induced increases in the levels of anti-oxidants. It is therefore indicated that NAMPT may have an anti-oxidant role in PC12 cells and may reduce the consumption of other anti-oxidants, including GSH and SOD. Furthermore, the levels of MDA, a biomarker of lipid oxidation, were markedly increased after 6-OHDA treatment further confirming that 6-OHDA exerts its neurotoxic effects by inducing oxidative stress in PC12 cells. However, pre-treatment with NMN significantly reduced MDA levels in PC12 cells following 6-OHDA treatment, further indicating its anti-oxidant effects. In brief, NAMPT could enhance the ability of scavenging anti-oxidant action and the free radicals in by 6-OHDA induced PD cell models.

SIRT1 is a unique protein deacetylator and is closely linked to cellular survival due to its dependence on $\mathrm{NAD}^{+}$(34). In the present study, it was demonstrated that 6-OHDA decreased SIRT1 activation in PC12 cells, which was attenuated by NMN. Furthermore, the neuroprotective compound resveratrol inhibited 6-OHDA-induced toxicity and increased the expression of SIRT1 in PC12 cells. These results indicated that NAMPT/NMN may exert their neuroprotective/anti-oxidant effects via activation of SIRT1.

In conclusion, the present study demonstrated that NAMPT, a rate-limiting enzyme for mammalian $\mathrm{NAD}^{+}$, markedly protected PC12 cells against 6-OHDA-induced oxidative stress-associated cell death. The protective effects of NAMPT/NMN may be attributed to, at least in part, their potent anti-oxidant properties, as evidenced by the marked increases in GSH and SOD as well as the reduction of MDA, through enhancing SIRT1 activity. NAMPT and NAD as well as SIRT1 may therefore have a crucial role in PD and other neurodegenerative disorders, and their upregulation may represent a novel therapeutic strategy.

\section{References}

1. Beal MF: Experimental models of Parkinson's disease. Nat Rev Neurosci 2: 325-334, 2001.

2. Hanrott K, Gudmunsen L, O'Neill MJ and Wonnacott S: 6-hydroxydopamine-induced apoptosis is mediated via extracellular auto-oxidation and caspase 3-dependent activation of protein kinase Cdelta. J Biol Chem 281: 5373-5382, 2006.

3. Blandini F, Armentero MT and Martignoni E: The 6-hydroxydopamine model: News from the past. Parkinsonism Relat Disord 14 (Suppl 2): S124-S129, 2008.

4. Surendran S and Rajasankar S: Parkinson's disease: Oxidative stress and therapeutic approaches. Neurol Sci 31: 531-540, 2010.

5. Soto-Otero R, Méndez-Alvarez E, Hermida-Ameijeiras A, Muñoz-Patiño AM and Labandeira-Garcia JL: Autoxidation and neurotoxicity of 6-hydroxydopamine in the presence of some antioxidants. J Neurochem 74: 1605-1612, 2000.
6. Han XH, Cheng MN, Chen L, Fang H, Wang LJ, Li XT and Qu ZQ: 7,8-dihydroxyflavone protects PC12 cells against 6-hydroxydopamine-induced cell death through modulating PI3K/Akt and JNK pathways. Neurosci Lett 581: 85-88, 2014.

7. Xu DP, Zhang K, Zhang ZJ, Sun YW, Guo BJ, Wang YQ, Hoi PM, Han YF and Lee SM: A novel tetramethylpyrazine bis-nitrone (TN-2) protects against 6-hydroxyldopamine-induced neurotoxicity via modulation of the NF- $\mathrm{KB}$ and the PKC $\alpha / \mathrm{PI} 3-\mathrm{K} / \mathrm{Akt}$ pathways. Neurochem Int 78: 76-85, 2014.

8. Wang L, Wang R, Jin M, Huang Y, Liu A, Qin J, Chen M, Wen S, Pi R and Shen W: Carvedilol attenuates 6-hydroxydopamine-induced cell death in PC12 Cells: Involvement of Akt and Nrf2/ARE pathways. Neurochem Res 39: 1733-1740, 2014.

9. Imai SI, Armstrong CM, Kaeberlein M and Guarente L: Transcriptional silencing and longevity protein Sir2 is an NAD-dependent histone deacetylase. Nature 403: 795-800, 2000.

10. Rongvaux A, Shea RJ, Mulks MH, Gigot D, Urbain J, Leo O and Andris F: Pre-B-cell colony-enhancing factor, whose expression is up-regulated in activated lymphocytes, is a nicotinamide phosphoribosyltransferase, a cytosolic enzyme involved in NAD biosynthesis. Eur J Immunol 32: 3225-3234, 2002.

11. Revollo JR, Grimm AA and Imai S: The regulation of nicotinamide adenine dinucleotide biosynthesis by Nampt/PBEF/visfatin in mammals. Curr Opin Gastroenterol 23: 164-170, 2007.

12. Revollo JR, Grimm AA and Imai S: The NAD biosynthesis pathway mediated by nicotinamide phosphoribosyltransferase regulates Sir2 activity in mammalian cells. J Biol Chem 279: 50754-50763, 2004.

13. Ying $\mathrm{W}: \mathrm{NAD}^{+} / \mathrm{NADH}$ and $\mathrm{NADP}+/ \mathrm{NADPH}$ in cellular functions and cell death: Regulation and biological consequences. Antioxid Redox Signal 10: 179-206, 2008.

14. Furukawa A, Tada-Oikawa S, Kawanishi S and Oikawa $\mathrm{S}: \mathrm{H}_{2} \mathrm{O}_{2}$ accelerates cellular senescence by accumulation of acetylated p53 via decrease in the function of SIRT1 by NAD ${ }^{+}$depletion. Cell Physiol Biochem 20: 45-54, 2007.

15. Lu L, Tang L, Wei W, Hong Y, Chen H, Ying W and Chen S: Nicotinamide mononucleotide improves energy activity and survival rate in an in vitro model of Parkinson's disease. Exp Ther Med 8: 943-950, 2014.

16. Milne JC, Lambert PD, Schenk S, Carney DP, Smith JJ, Gagne DJ, Jin L, Boss O, Perni RB, Vu CB, et al: Small molecule activators of SIRT1 as therapeutics for the treatment of type 2 diabetes. Nature 450: 712-716, 2007.

17. Araki T, Sasaki Y and Milbrandt J: Increased nuclear NAD biosynthesis and SIRT1 activation prevent axonal degeneration. Science 305: 1010-1013, 2004.

18. Tanno M, Kuno A, Yano T, Miura T, Hisahara S, Ishikawa S, Shimamoto K and Horio Y: Induction of manganese superoxide dismutase by nuclear translocation and activation of SIRT1 promotes cell survival in chronic heart failure. J Biol Chem 285: 8375-8382, 2010.

19. Liu T, Liu PY and Marshall GM: The critical role of the class III histone deacetylase SIRT1 in cancer. Cancer Res 69: 1702-1705, 2009.

20. Cohen HY, Miller C, Bitterman KJ, Wall NR, Hekking B, Kessler B, Howitz KT, Gorospe M, de Cabo R and Sinclair DA: Calorie restriction promotes mammalian cell survival by inducing the SIRT1 deacetylase. Science 305: 390-392, 2004.

21. Salminen A, Huuskonen J, Ojala J, Kauppinen A, Kaarniranta K and Suuronen T: Activation of innate immunity system during aging: NF-kB signaling is the molecular culprit of inflamm-aging. Ageing Res Rev 7: 83-105, 2008.

22. Chen WY, Wang DH, Yen RC, Luo J, Gu W and Baylin SB: Tumor suppressor HIC1 directly regulates SIRT1 to modulate p53-dependent DNA-damage responses. Cell 123: 437-448, 2005.

23. Hasegawa K, Wakino S, Yoshioka K, Tatematsu S, Hara Y, Minakuchi H, Washida N, Tokuyama H, Hayashi K and Itoh H: Sirt1 protects against oxidative stress-induced renal tubular cell apoptosis by the bidirectional regulation of catalase expression. Biochem Biophys Res Commun 372: 51-56, 2008.

24. Jeong J, Juhn K, Lee H, Kim SH, Min BH, Lee KM, Cho MH, Park GH and Lee KH: SIRT1 promotes DNA repair activity and deacetylation of Ku70. Exp Mol Med 39: 8-13, 2007.

25. Bu Q, Yang Y, Yan G, Hu Z, Hu C, Duan J, Lv L, Zhou J, Zhao J, Shao X, et al: Proteomic analysis of the nucleus accumbens in rhesus monkeys of morphine dependence and withdrawal intervention. J Proteomics 75: 1330-1342, 2012. 
26. Zhao X, Zhai S, An MS, Wang YH, Yang YF, Ge HQ, Liu JH and Pu XP: Neuroprotective effects of protocatechuic aldehyde against neurotoxin-induced cellular and animal models of Parkinson's disease. PloS One 8: e78220, 2013.

27. Blum D, Torch S, Lambeng N, Nissou M, Benabid AL, Sadoul R and Verna JM: Molecular pathways involved in the neurotoxicity of 6-OHDA, dopamine and MPTP: Contribution to the apoptotic theory in Parkinson's disease. Prog Neurobiol 65: 135-172, 2001.

28. Schober A: Classic toxin-induced animal models of Parkinson's disease: 6-OHDA and MPTP. Cell Tissue Res 318: 215-224, 2004.

29. Saito Y, Nishio K, Ogawa Y, Kinumi T, Yoshida Y, Masuo Y and Niki E: Molecular mechanisms of 6-hydroxydopamine-induced cytotoxicity in PC12 cells: Involvement of hydrogen peroxide-dependent and-independent action. Free Radic Biol Med 42: 675-685, 2007
30. Fujita H, Ogino T, Kobuchi H, Fujiwara T, Yano H, Akiyama J, Utsumi $\mathrm{K}$ and Sasaki J: Cell-permeable cAMP analog suppresses 6-hydroxydopamine-induced apoptosis in PC12 cells through the activation of the Akt pathway. Brain Res 1113: 10-23, 2006.

31. Yang T and Sauve AA: NAD metabolism and sirtuins: Metabolic regulation of protein deacetylation in stress and toxicity. AAPS J 8: E632-E643, 2006.

32. Bürkle A: Poly (ADP-ribose). The most elaborate metabolite of NAD $^{+}$. FEBS J 272: 4576-4589, 2005.

33. Halliwell B: Oxidative stress and neurodegeneration: Where are we now? J Neurochem 97: 1634-1658, 2006.

34. Zhang T and Kraus WL: SIRT1-dependent regulation of chromatin and transcription: Linking NAD metabolism and signaling to the control of cellular functions. Biochim Biophys Acta 1804: 1666-1675, 2010. 